\title{
Identifying the pattern of immune related cells and genes in the peripheral blood of ischemic stroke
}

\author{
Zijian Li, Yueran Cui, Juan Feng and Yanxia Guo*
}

\begin{abstract}
Background: Ischemic stroke (IS) is the second leading cause of death worldwide which is a serious hazard to human health. Evidence suggests that the immune system plays a key role in the pathophysiology of IS. However, the precisely immune related mechanisms were still not been systematically understood.

Methods: In this study, we aim to identify the immune related modules and genes that might play vital role in the occurrence and development of IS by using the weighted gene co-expression network analysis (WGCNA). Meanwhile, we applied a kind of deconvolution algorithm to reveal the proportions of 22 subsets of immune cells in the blood samples.

Results: There were total 128 IS patients and 67 healthy control samples in the three Gene Expression Omnibus (GEO) datasets. Under the screening criteria, 1082 DEGs (894 up-regulated and 188 down-regulated) were chosen for further analysis. A total of 11 clinically significant modules were identified, from which immune-related hub modules and hub genes were further explored. Finally, 16 genes were selected as real hub genes for further validation analysis. Furthermore, these CIBERSORT results suggest that detailed analysis of the immune subtype distribution pattern has the potential to enhance clinical prediction and to identify candidates for immunotherapy. More specifically, we identified that neutrophil emerge as a promising target for IS therapies.
\end{abstract}

Conclusions: In the present study, we investigated the immune related gene expression modules, in which the SLAMF1, IL7R and NCF4 may be novel therapeutic targets to promote functional and histological recovery after ischemic stroke. Furthermore, these hub genes and neutrophils may become important biological targets in the drug screening and drug designing.

Keywords: Ischemic stroke, Weighted gene co-expression network analysis, Bioinformatics analysis, Immune cell subtype distribution pattern, Pathological process

\section{Background}

Stroke is one of the leading causes of death and disability in the world, of which ischemic stroke accounts for more than $80 \%$. The burden of ischemic stroke is increasing rapidly with the acceleration of social aging

*Correspondence: yxguoneurology@163.com

Department of Neurology, Shengjing Hospital of China Medical University, No. 36 Sanhao Street, Heping District, Shenyang 110004, Liaoning, People's Republic of China and urbanization, the prevalence of unhealthy lifestyles, the exposure of cardiovascular risk factors [1]. Therefore, the early diagnosis and treatment of IS are faced with great challenges, which need to be further strengthened. During the development of stroke, inflammatory cell infiltration can stimulate intense immune response and cause dysfunction of immune microenvironment in central nervous system, which may further exacerbate the disease [2]. At present, some researches indicate that immunoregulation can delay disease progression as well 
as improve neurological function and prognosis, which further emphasize the importance of restoring immune microenvironmental homeostasis in the central nervous system [3, 4]. Therefore, in addition to conventional treatment, immunoregulatory therapy as an alternative treatment method is worthy of in-depth study. However, there are limited systematic studies on immune-related gene modules and peripheral blood immune cell subtype distribution patterns in stroke patients.

With the extensive applications and constant development of the gene chip technology, weighted gene coexpression network analysis is increasing used to analysis huge amounts of gene expression data, which is a powerful systematic biological method to analyze the molecular mechanism and network relationship [5]. WGCNA is used to look for co-expressed gene modules, to explore associations between gene networks and interesting sample characteristics, such as health and disease samples, as well as hub genes in the network [6]. In this study, we used the differentially expressed genes between ischemic stroke patients and healthy control in three GEO datasets to construct a co-expression network by WGCNA. We attempted to identify promising immune related candidate biomarkers or potential therapeutic targets of IS from modules in which highly correlated genes clustered. Furthermore, the study of specific molecular and biological functions of these hub genes may better for understanding the underlying mechanisms of IS.

Recent years, a computational analytical tool named CIBERSORT was established to provide an estimation of the abundances of member cell types in a mixed cell population, using gene expression microarray data $[7,8]$. By applying CIBERSORT, we assessed the relative proportions of immune cells in 23 blood samples of healthy people and 69 blood samples of ischemic patients from GSE58294 dataset. The obtained immune cell profiles provided proportion and activation status of 22 immune cell subtypes.
In this study, the analysis of the differentially expressed genes between IS patients and healthy people is conducive to revealing the immune related signal pathway mechanism, finding effective targets for the treatment of IS, and laying a foundation for the development of immunoregulatory treatment programs for IS.

\section{Materials and methods \\ Microarray datasets collection}

The GEO database (http://www.ncbi.nlm.nih.gov/ geo) was used to obtain the gene expression profiles of ischemic stroke. The inclusion criteria were the following: (1) the whole-genome expression profiling of whole blood or peripheral mononuclear cells of IS patients or healthy control samples were available in the datasets; (2) every included dataset contains no less than 20 stroke samples and/or 20 healthy control samples. The exclusion criteria of datasets in this study were set as follows: (1) the IS patients which received any treatment before collection of samples; (2) the control samples were people with definite risk of cardiovascular and cerebrovascular events. The gene expression profiles of stroke GSE58294, GSE22255, GSE16561 and GSE37587 were downloaded. The details of these microarray data were listed in the Table 1.

\section{Data preprocessing and study design}

The series matrix of each expression datasets was obtained from GEO database. R software (version 3.5.1) was used to perform the bioinformatics analysis [9]. The "affy" package in $\mathrm{R}$ was used to conduct the normalization and background correction of data. Then, the probe level data were then converted into gene expression values. For multiple probes corresponding to a gene, the average expression value was taken as the gene expression value. The difference between batches were eliminated of total 195 samples including 128 stroke patient samples and 67 healthy control samples by ComBat in the

\section{Table 1 Basic information of gene expression profiling}

\begin{tabular}{|c|c|c|c|c|c|c|c|}
\hline GEO Accession ID & Platform & Samples (Total number) & Number of cases & Number of controls & Country & Year & Author \\
\hline \multicolumn{8}{|l|}{ Training set } \\
\hline GSE58294 & GPL570 & Whole blood samples (92) & $\begin{array}{l}69 \text { ischemic stroke } \\
\text { samples }\end{array}$ & 23 controls & USA & 2014 & Boryana Stamova \\
\hline GSE22255 & GPL570 & $\begin{array}{l}\text { Peripheral blood mono- } \\
\text { nuclear cells (40) }\end{array}$ & $\begin{array}{l}20 \text { ischemic stroke } \\
\text { samples }\end{array}$ & $\begin{array}{l}20 \text { sex- and age-matched } \\
\text { controls }\end{array}$ & Portugal & 2011 & Sofia A Oliveira \\
\hline GSE16561 & GPL6883 & Whole blood samples (63) & $\begin{array}{l}39 \text { ischemic stroke } \\
\text { samples }\end{array}$ & 24 healthy controls & USA & 2010 & Taura L. Barr \\
\hline \multicolumn{8}{|l|}{ Validation set } \\
\hline GSE37587 & GPL6883 & Whole blood samples (68) & $\begin{array}{l}68 \text { ischemic stroke } \\
\text { samples }\end{array}$ & - & USA & 2015 & Taura L. Barr \\
\hline
\end{tabular}


"sva" R package. The distribution patterns of disease and control samples (before and after batch correction with ComBat) was observed by principal component analysis (PCA). The flow diagram of this study is shown in Additional file 1: Figure S1.

\section{Identification of differentially expressed genes in IS}

The "limma" package in $\mathrm{R}$ was used to obtained differentially Expressed Genes (DEGs) between the stroke patient samples and the healthy control samples in the expressing data. Then we carried out the significance analysis of microarrays and set the select criteria as false discovery rate (FDR) value $<0.05$ and Fold change $>1.2$ for further network construction.

\section{Construction of co-expression network}

The "WGCNA" package in $\mathrm{R}$ was used to construct the co-expression network based on the expression data profile of DEGs. The microarray quality was checked by the "impute" package in $\mathrm{R}$ which could detect whether the genes had missing values, and ensure they were good samples. We performed sample clustering to plot the sample tree and detect outliers. Then, we performed Pearson's correlation matrices for pair-wise genes and found the soft thresholding power $\beta$ value by using the pickSoft Threshold function of WGCNA.

\section{Gene Ontology (GO) and Kyoto encyclopedia of genes and genomes (KEGG) analysis}

GO Enrichment and KEGG pathway analysis of all genes in hub modules were performed by using the online database STRING (Search Tool for the retrieval of Interacting Genes/Proteins). Furthermore, the PPI network was construct by STRING and Cytoscape 3.7.1.

\section{Identification of hub genes}

First, we defined hub modules as module in which the absolute value of the Pearson's correlation of module membership $>0.2$ and p-value $<0.05$. Furthermore, we defined hub genes in co-expression network as genes both satisfies two conditions: the absolute value of the Pearson's correlation of module membership $>0.8$ and the absolute value of the Pearson's correlation of gene trait relationship $>0.2$, which represented high module connectivity and high clinical significance, respectively. Genes in the hub modules were analyzed to construct a protein-protein interaction (PPI) network, and we defined hub genes in PPI network as genes with a connectivity degree (set default filter as degree in and out) of $>8$. Then, we obtained the real hub genes by taking the intersection of hub genes in co-expression network and hub genes in PPI network.

\section{Evaluation of immune cell subtype distribution}

CIBERSORT is a kind of deconvolution algorithm, by which the normalized gene expression matrix can be transformed into the composition of infiltrating immune cells. During the CIBERSORT calculating, the abundance of specific cell types in complex tissue was quantified, and the results of CIBERSORT have been validated by fluorescence activated cell sorting (FACS). LM22 was used as a reference expression signature with 1000 permutations. More accurately forecast of immune cell composition was defined as the CIBERSORT output of $\mathrm{p}$-value $<0.05$. Then samples satisfying constraint were selected for further analysis. In this study, the dataset GSE58294 including 69 ischemic stroke samples and 23 healthy control samples were used to estimate the infiltrated immune cells at the time of the stroke. The 22 kinds of infiltrated immune cells included B cells (naïve B cells and memory B cells), $\mathrm{T}$ cells $\left(\mathrm{CD}^{+}{ }^{+} \mathrm{T}\right.$ cells, naïve $\mathrm{CD} 4^{+} \mathrm{T}$ cells, memory resting $\mathrm{CD}^{+}{ }^{+} \mathrm{T}$ cells, memory activated $\mathrm{CD} 4^{+} \mathrm{T}$ cells, follicular helper T cells, regulatory T cells and $\gamma \delta \mathrm{T}$ cells), NK cells (resting NK cells and activated NK cells), monocytes, macrophages (M0 macrophages, M1 macrophages, M2 macrophages), dendritic cells (resting dendritic cells and activated dendritic cells), mast cells (resting mast cells and activated mast cells), eosinophils and neutrophils. All evaluated 22 kinds of immune cell type fractions were to sum up to 1 for each sample.

\section{Validation of hub genes by microarray dataset}

The dataset GSE37587 including 68 stroke samples was used for the validation of the real hub genes. The validation samples were composed by stroke samples of GSE375887 and healthy control samples of GSE16561. We examined the expression of real hub genes in these samples. Differences were considered statistically significant at $\mathrm{p}$-value $<0.05$.

\section{Sample collection and blood routine examination}

This study was approved by the ethical committee of Shengjing Hospital, China Medical University (No. 2017PS161K). We obtained informed consent from all participating individuals. The peripheral blood samples were obtained from fifteen patients with ischemic stroke. Fifteen healthy individuals were used as control samples. Clinical characteristics of these samples were listed in Additional file 2: Table S2. Ten milliliters peripheral blood were divided into equal amounts for blood routine examination and quantitative real time polymerase chain reaction, respectively. Blood routine examination was determined by COULTER GEN.S blood cell five-classification analyzer, and 
blood ingredients (especially neutrophil percentage) were measured by VCS (volume, conductivity, scatter) methods.

\section{Quantitative real time polymerase chain reaction (qRT-PCR)}

Subsequently, qRT-PCR was used to verify the expression of the 16 hub genes in peripheral blood of clinical samples. Total RNA was isolated from each sample using Takara RNAiso Plus (9108) Trizol reagent according to the manufacturer's instructions. Reverse transcription from total RNA to cDNA and qRT-PCR were performed using the Takara PrimeScript RT Master Mix (RR036A) and SYBR Green Premix (RR420A), respectively. The results were analyzed using the $2-\Delta \Delta \mathrm{Ct}$ method and represented as fold changes, normalized to GAPDH. The PCR primers used in this study were shown in Additional file 2: Table S1. Statistically significant was considered as the p-value $<0.05$.

\section{Results}

Data preprocessing and identification of DEGs

After batch correction with ComBat, the scatter plot of PCA shown that two significantly different distribution patterns between ischemic stroke patient and healthy control samples. Samples of ischemic stroke were mainly distributed on the left side of plot, while healthy control samples were mainly distributed on the right side, as shown in Fig. 1b.

The expression matrixes of three GEO datasets including 128 IS patients and 67 healthy control samples downloaded. Samples of GSE58294, GSE22255 and GSE16561 were merged into one training data set which containing a total of 13,250 genes. After data merge and eliminate

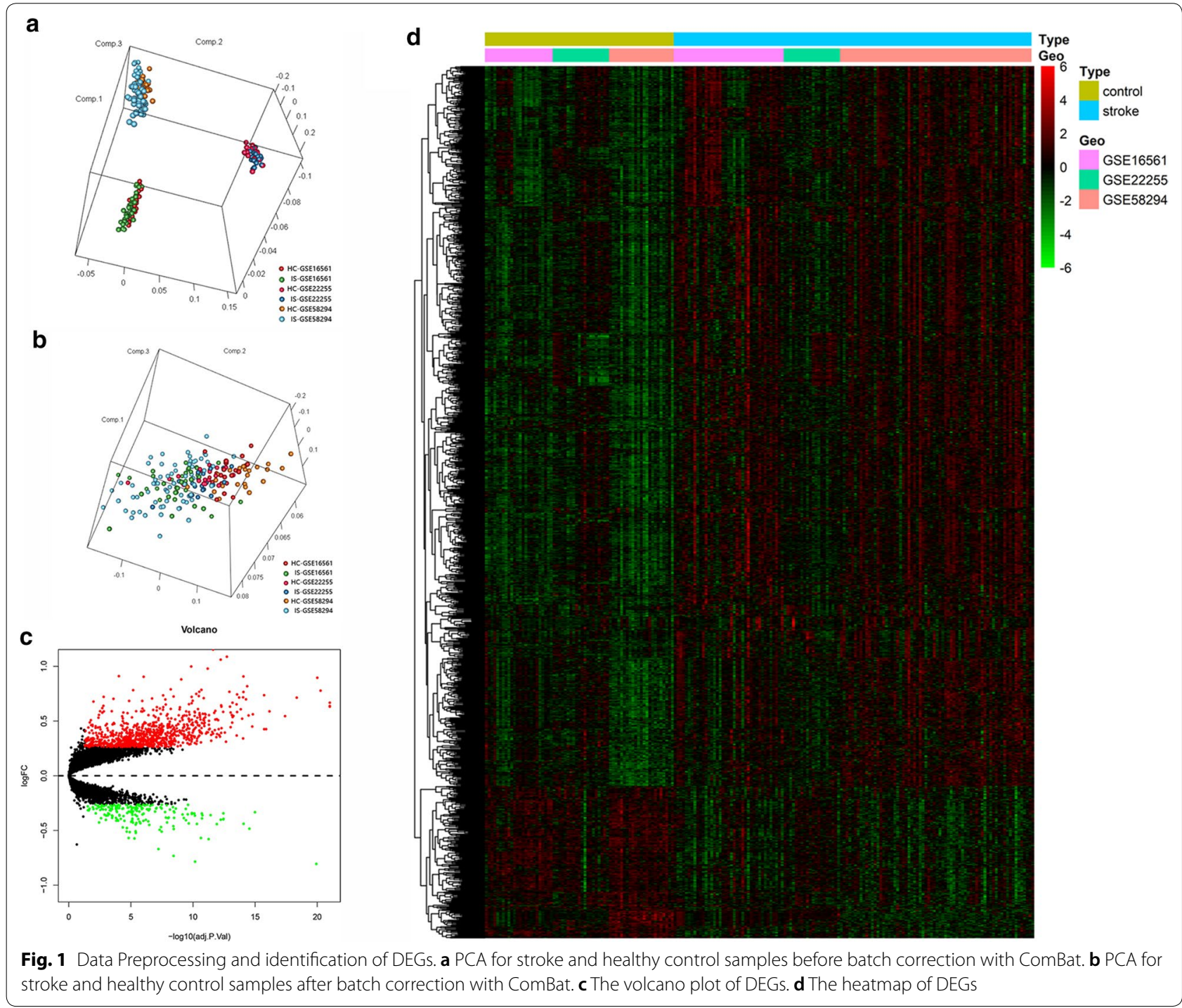


the difference between batch, we obtained the expression matrices from the 195 samples in training dataset. Under the threshold of FDR $<0.05$ and fold change $>1.2$, a total of 1082 DEGs (894 up-regulated and 188 downregulated) were chosen for further analysis. The volcano plot and heatmap of DEGs were shown in Fig. 1c, d. The expression matrices of total 1082 DEGs in training set were showed in Additional file 3.

Training set quality assessment and co-expression network construction

As shown in Additional file 4: Figure S2A, seven samples (GSM416554, GSM1406037, GSM1406051, GSM1406046, GSM1406045, GSM1406041,
GSM1406055) were removed as outliers from subsequent analysis. Then, we matched the disease state of samples with their expression matrixes. The remaining $188 \mathrm{sam}$ ples were re-clustered and the sample dendrogram and trait heatmap were plotted (Additional file 4: Figure S2B). In this study, the soft-thresholding powers was chosen as $\beta=8$ where the curve first reached $R^{2}=0.79$, to construct a weighted network based on a scale-free topology criterion (Additional file 5: Figure S3A-D).

\section{Identification of clinically significant modules}

A total of 11 modules were identified through the dynamic tree cutting method, as shown in Fig. 2a. And the number of genes in each module was listed in Table 2.
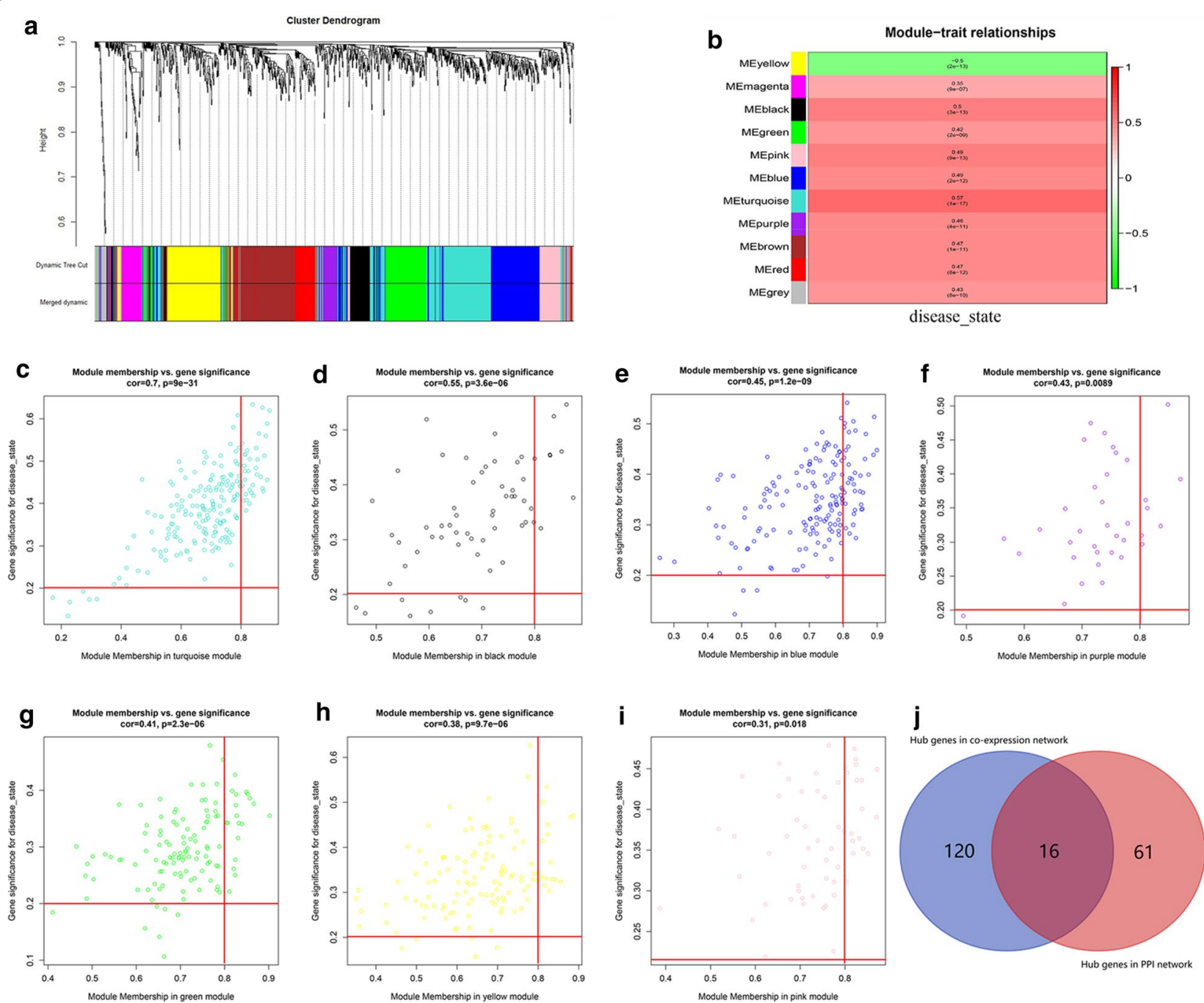

Fig. 2 Cluster dendrogram and module-trait relationship plot. a Dendrogram of all differentially expressed genes clustered based on a dissimilarity measure (1-TOM). b Heatmap of the correlation between module eigengenes and ischemic stroke. Scatter diagrams for module membership vs. gene significance of disease state. c Turquoise module. $\mathbf{d}$ Black module. e Blue module. f Purple module. $\mathbf{g}$ Green module. $\mathbf{h}$ Yellow module. i Pink module. $\mathbf{j}$ Venn plot of hub genes in the co-expression and PPI networks 
Table 2 The number of genes in the 11 modules

\begin{tabular}{lc}
\hline Module colors & Number \\
\hline Black & 62 \\
Blue & 166 \\
Brown & 152 \\
Green & 124 \\
Grey & 43 \\
Magenta & 47 \\
Pink & 58 \\
Purple & 36 \\
Red & 66 \\
Turquoise & 200 \\
Yellow & 128 \\
\hline
\end{tabular}

After relating modules to traits, high correlations were observed in the trait of disease state (healthy control or ischemic stroke), as shown in Fig. 2b. Clinically significant modules were considered as the p-value $<0.05$ and eligible for further analysis, which included turquoise module, black module, blue module, purple module, green module, yellow module and pink module.

\section{Identification of hub genes}

136 hub genes were selected from the clinically significant modules in the co-expression network by WGCNA (Fig. 2c-i and Additional file 6: Figure S5), and 77 genes were selected through PPI network analysis (Additional file 7: Figure S4A-D and Additional file 8: Figure S6). As listed in Table 3, 16 hub genes in both the co-expression and PPI networks were selected as real hub genes for further validation analysis (Fig. 2j).

\section{Functional enrichment annotation}

To further explore the function of each module, we performed the GO and KEGG pathway analysis. The GO analysis results showed that genes in hub modules were mainly enriched in immune response, immune system process, immune effector process, neutrophil degranulation and leukocyte activation (terms shared by three or more modules). The detailed top-ten GO function annotation terms of each module were selected and listed in the Table 4 (according to p-value). The KEGG pathways of each module were listed in Table 5, which mainly enriched in pathways of Complement and coagulation cascades, NOD-like receptor signaling pathway, Th17 cell differentiation, Th1 and Th2 cell differentiation and $\mathrm{T}$ cell receptor signaling pathway and so on. Most of these

Table 3 The real hub genes in the co-expression and PPI networks

\begin{tabular}{|c|c|c|c|c|c|}
\hline GeneSymbol & ModuleColor & GS.disease state & p.GS. disease state & MM.of related module & $\begin{array}{l}\text { p.MM.of } \\
\text { related } \\
\text { module }\end{array}$ \\
\hline TLR8 & Turquoise & 0.435661339 & $4.14 \mathrm{E}-10$ & 0.871990002 & $1.33 E-59$ \\
\hline PTEN & Turquoise & 0.428731523 & $8.34 \mathrm{E}-10$ & 0.874600844 & $2.22 \mathrm{E}-60$ \\
\hline IFNAR1 & Turquoise & 0.407160397 & $6.68 \mathrm{E}-09$ & 0.818666737 & $1.12 \mathrm{E}-46$ \\
\hline CCR7 & Yellow & -0.41442059 & 3.37E-09 & 0.810615379 & $4.24 \mathrm{E}-45$ \\
\hline IL7R & Yellow & -0.31537227 & $1.04 \mathrm{E}-05$ & 0.816302419 & $3.31 E-46$ \\
\hline SLAMF1 & Yellow & -0.333846288 & $2.84 \mathrm{E}-06$ & 0.808174198 & $1.23 E-44$ \\
\hline TIMP2 & Blue & 0.447586714 & $1.20 \mathrm{E}-10$ & 0.899305925 & $1.00 \mathrm{E}-68$ \\
\hline CANT1 & Blue & 0.390141685 & $3.12 \mathrm{E}-08$ & 0.82537424 & $4.68 \mathrm{E}-48$ \\
\hline PAK1 & Blue & 0.501249842 & $2.36 \mathrm{E}-13$ & 0.804602912 & $5.74 \mathrm{E}-44$ \\
\hline ATG7 & Blue & 0.432671718 & $5.61 \mathrm{E}-10$ & 0.802242306 & $1.56 E-43$ \\
\hline NCF4 & Blue & 0.293300473 & $4.41 \mathrm{E}-05$ & 0.807809113 & $1.45 E-44$ \\
\hline ITGAM & Blue & 0.541718688 & $9.94 \mathrm{E}-16$ & 0.811555078 & $2.80 E-45$ \\
\hline WAS & Blue & 0.479687326 & $3.29 E-12$ & 0.866722618 & 4.37E-58 \\
\hline MYD88 & Blue & 0.447728134 & $1.18 \mathrm{E}-10$ & 0.80773727 & $1.49 E-44$ \\
\hline MAPK1 & Blue & 0.430201337 & $7.20 \mathrm{E}-10$ & 0.864080663 & $2.39 E-57$ \\
\hline FGR & Blue & 0.402714638 & $1.01 \mathrm{E}-08$ & 0.834484479 & $5.05 E-50$ \\
\hline
\end{tabular}

TLR toll like receptor 8, PTEN phosphatase and tensin homolog, IFNAR1 interferon alpha and beta receptor subunit 1, CCR7 C-C motif chemokine receptor 7, IL7R interleukin 7 receptor, SLAMF1 signaling lymphocytic activation molecule family member 1, TIMP2 tissue inhibitor of metalloproteinases-2, CANT1 calcium activated nucleotidase 1, PAK1 p21 (RAC1) activated kinase 1, ATG7 autophagy related 7, NCF4 neutrophil cytosolic factor 4, ITGAM integrin subunit alpha M, WAS WASP actin nucleation promoting factor, MYD88 MYD88 innate immune signal transduction adaptor, MAPK1 mitogen-activated protein kinase 1, FGR proto-oncogene, Src family tyrosine kinase, FGR 
Table 4 The top $10 \mathrm{GO}$ enrichment terms of genes in each module

\begin{tabular}{|c|c|c|c|c|}
\hline GO term ID & Term description & $\begin{array}{l}\text { Observed gene } \\
\text { count }\end{array}$ & $\begin{array}{l}\text { Background gene } \\
\text { count }\end{array}$ & False discovery rate \\
\hline \multicolumn{5}{|l|}{ Black module } \\
\hline GO:0002376 & Immune system process & 29 & 2370 & 4.39E-08 \\
\hline GO:0006955 & Immune response & 24 & 1560 & $4.39 \mathrm{E}-08$ \\
\hline GO:0002237 & Response to molecule of bacterial origin & 12 & 317 & $3.03 E-07$ \\
\hline GO:0071345 & Cellular response to cytokine stimulus & 18 & 953 & $3.5 \mathrm{E}-07$ \\
\hline GO:0032496 & Response to lipopolysaccharide & 11 & 298 & $1.13 \mathrm{E}-06$ \\
\hline GO:0019221 & Cytokine-mediated signaling pathway & 14 & 655 & $5.05 \mathrm{E}-06$ \\
\hline GO:0009617 & Response to bacterium & 13 & 555 & $5.46 \mathrm{E}-06$ \\
\hline GO:0010033 & Response to organic substance & 27 & 2815 & 7.47E-06 \\
\hline GO:0007166 & Cell surface receptor signaling pathway & 23 & 2198 & $2.47 \mathrm{E}-05$ \\
\hline GO:0071310 & Cellular response to organic substance & 23 & 2219 & $2.67 \mathrm{E}-05$ \\
\hline \multicolumn{5}{|l|}{ Blue module } \\
\hline GO:0043312 & Neutrophil degranulation & 45 & 485 & $4.58 \mathrm{E}-29$ \\
\hline GO:0002366 & Leukocyte activation involved in immune response & 47 & 616 & $5.58 \mathrm{E}-28$ \\
\hline GO:0045055 & Regulated exocytosis & 48 & 691 & $4.66 \mathrm{E}-27$ \\
\hline GO:0006887 & Exocytosis & 50 & 774 & $5.35 \mathrm{E}-27$ \\
\hline GO:0002443 & Leukocyte mediated immunity & 46 & 632 & $1.14 \mathrm{E}-26$ \\
\hline GO:0006955 & Immune response & 65 & 1560 & $6.26 \mathrm{E}-26$ \\
\hline GO:0002252 & Immune effector process & 52 & 927 & $1.49 \mathrm{E}-25$ \\
\hline GO:0045321 & Leukocyte activation & 50 & 894 & $2.16 \mathrm{E}-24$ \\
\hline GO:0001775 & Cell activation & 51 & 1024 & $8.55 E-23$ \\
\hline GO:0016192 & Vesicle-mediated transport & 63 & 1699 & $2.08 \mathrm{E}-22$ \\
\hline \multicolumn{5}{|l|}{ Green module } \\
\hline GO:0002252 & Immune effector process & 19 & 927 & 0.0071 \\
\hline GO:0002274 & Myeloid leukocyte activation & 15 & 574 & 0.0071 \\
\hline GO:0002443 & Leukocyte mediated immunity & 16 & 632 & 0.0071 \\
\hline GO:0001775 & Cell activation & 19 & 1024 & 0.009 \\
\hline GO:0002366 & Leukocyte activation involved in immune response & 14 & 616 & 0.009 \\
\hline GO:0002697 & Regulation of immune effector process & 11 & 362 & 0.009 \\
\hline GO:0043312 & Neutrophil degranulation & 13 & 485 & 0.009 \\
\hline GO:0045321 & Leukocyte activation & 18 & 894 & 0.009 \\
\hline GO:0006810 & Transport & 44 & 4130 & 0.0163 \\
\hline GO:0006887 & Exocytosis & 15 & 774 & 0.0163 \\
\hline \multicolumn{5}{|l|}{ Pink module } \\
\hline GO:0051345 & Positive regulation of hydrolase activity & 12 & 742 & 0.0037 \\
\hline GO:0001932 & Regulation of protein phosphorylation & 12 & 1370 & 0.021 \\
\hline GO:0001933 & Negative regulation of protein phosphorylation & 8 & 422 & 0.021 \\
\hline GO:0002252 & Immune effector process & 10 & 927 & 0.021 \\
\hline GO:0002275 & Myeloid cell activation involved in immune response & 9 & 519 & 0.021 \\
\hline GO:0002376 & Immune system process & 18 & 2370 & 0.021 \\
\hline GO:0002444 & Myeloid leukocyte mediated immunity & 9 & 519 & 0.021 \\
\hline GO:0006334 & Nucleosome assembly & 5 & 137 & 0.021 \\
\hline GO:0006810 & Transport & 24 & 4130 & 0.021 \\
\hline GO:0006955 & Immune response & 15 & 1560 & 0.021 \\
\hline \multicolumn{5}{|l|}{ Purple module } \\
\hline GO:0043312 & Neutrophil degranulation & 11 & 485 & $8.96 \mathrm{E}-07$ \\
\hline GO:0006952 & Defense response & 14 & 1234 & $1.28 \mathrm{E}-06$ \\
\hline GO:0002376 & Immune system process & 18 & 2370 & 2.07E-06 \\
\hline GO:0001819 & Positive regulation of cytokine production & 9 & 390 & $2.31 \mathrm{E}-06$ \\
\hline
\end{tabular}


Table 4 (continued)

\begin{tabular}{|c|c|c|c|c|}
\hline GO term ID & Term description & $\begin{array}{l}\text { Observed gene } \\
\text { count }\end{array}$ & $\begin{array}{l}\text { Background gene } \\
\text { count }\end{array}$ & False discovery rate \\
\hline GO:0006955 & Immune response & 15 & 1560 & $2.31 \mathrm{E}-06$ \\
\hline GO:0045321 & Leukocyte activation & 12 & 894 & $2.33 \mathrm{E}-06$ \\
\hline GO:0032940 & Secretion by cell & 12 & 959 & 4.74E-06 \\
\hline GO:0016192 & Vesicle-mediated transport & 15 & 1699 & $5.49 \mathrm{E}-06$ \\
\hline GO:0006950 & Response to stress & 18 & 3267 & 0.00015 \\
\hline GO:0009056 & Catabolic process & 13 & 1859 & 0.00059 \\
\hline \multicolumn{5}{|c|}{ Turquoise module } \\
\hline GO:0006955 & Immune response & 58 & 1560 & $1.55 E-14$ \\
\hline GO:0002376 & Immune system process & 71 & 2370 & $3.57 \mathrm{E}-14$ \\
\hline GO:0002252 & Immune effector process & 42 & 927 & $8.96 E-13$ \\
\hline GO:0045087 & Innate immune response & 36 & 676 & $1 E-12$ \\
\hline GO:0006952 & Defense response & 46 & 1234 & $1.89 E-11$ \\
\hline GO:0050896 & Response to stimulus & 128 & 7824 & 4.85E-09 \\
\hline GO:0006950 & Response to stress & 73 & 3267 & $9.15 E-09$ \\
\hline GO:0080134 & Regulation of response to stress & 41 & 1299 & $6.2 \mathrm{E}-08$ \\
\hline GO:0034097 & Response to cytokine & 36 & 1035 & $7.1 \mathrm{E}-08$ \\
\hline GO:0048584 & Positive regulation of response to stimulus & 53 & 2054 & $8.11 \mathrm{E}-08$ \\
\hline \multicolumn{5}{|l|}{ Yellow module } \\
\hline GO:0002684 & Positive regulation of immune system process & 23 & 882 & 4.91E-05 \\
\hline GO:0002250 & Adaptive immune response & 13 & 280 & $8.36 \mathrm{E}-05$ \\
\hline GO:0008284 & Positive regulation of cell population proliferation & 22 & 878 & $8.36 \mathrm{E}-05$ \\
\hline GO:0007166 & Cell surface receptor signaling pathway & 36 & 2198 & 0.00011 \\
\hline GO:0050851 & Antigen receptor-mediated signaling pathway & 9 & 122 & 0.00011 \\
\hline GO:0048584 & Positive regulation of response to stimulus & 34 & 2054 & 0.00014 \\
\hline GO:0048583 & Regulation of response to stimulus & 50 & 3882 & 0.00019 \\
\hline GO:0002376 & Immune system process & 36 & 2370 & 0.00031 \\
\hline GO:0006955 & Immune response & 28 & 1560 & 0.00031 \\
\hline GO:0046649 & Lymphocyte activation & 13 & 358 & 0.00031 \\
\hline
\end{tabular}

pathways were associated with immune and inflammatory responses.

The results of the GO and KEGG pathway analysis confirmed that modules listed below, especially blue module, turquoise module and yellow module may play key roles in the immune system, such as immune response, immune cell activation and differentiation.

\section{Verification of hub genes using the dataset}

The validation cohort was made up of stroke patient samples in GSE37587 and healthy control samples in GSE16561. The two data sets used the same chip on the same platform, making the data merge reasonable. After the difference between batches were eliminated, standardized data was used for the validation of 16 hub genes. As shown in Fig. 3a-p, the validation results of total 16 genes were consistent with the multiple chips conjoint analysis. We found ITGAM, NCF4, PAK1,
PTEN, MYD88, FGR, TLR8, ATG7, MAPK1, IFNAR1, TIMP2, CANT1 and WAS were upregulated in IS samples and IL7R, SLAMF1 and CCR7 were downregulated in IS samples.

\section{Profile of immune cell subtype distribution pattern}

As shown in Fig. 4a, the histogram shows the general distribution of various immune cells in each sample. Different colors represent different types of immune cells. The height of each color represents the percentage of such cells in the sample, and the sum of the percentage of various immune cells is 1 . It was observed that neutrophils, resting mast cells, M1 and M2 macrophages, monocytes, $\mathrm{CD} 8^{+} \mathrm{T}$ cells, activated memory $C D 4^{+} \mathrm{T}$ cells, $\gamma \delta \mathrm{T}$ cells and naïve B cells are the main infiltrating cells. The distribution of some immune cell subsets with low abundance expression in stroke has not been fully revealed, due to the limitations of CIBERSORT algorithm. Proportions of immune cells from 
Table 5 The KEGG pathway enrichment analysis of genes in each module

\begin{tabular}{|c|c|c|}
\hline KEGG term ID & Term description & FDR \\
\hline \multicolumn{3}{|l|}{ Black module } \\
\hline hsa04668 & TNF signaling pathway & 0.0029 \\
\hline hsa04610 & Complement and coagulation cascades & 0.0065 \\
\hline hsa04621 & NOD-like receptor signaling pathway & 0.0068 \\
\hline \multicolumn{3}{|c|}{ Blue module (top 10) } \\
\hline hsa04142 & Lysosome & 0.0015 \\
\hline hsa04670 & Leukocyte transendothelial migration & 0.0015 \\
\hline hsa04380 & Osteoclast differentiation & 0.0078 \\
\hline hsa05152 & Tuberculosis & 0.0078 \\
\hline hsa00052 & Galactose metabolism & 0.0079 \\
\hline hsa05140 & Leishmaniasis & 0.0136 \\
\hline hsa04140 & Autophagy-animal & 0.0233 \\
\hline hsa04666 & Fc gamma R-mediated phagocytosis & 0.0286 \\
\hline hsa05161 & Hepatitis B & 0.032 \\
\hline hsa05322 & Systemic lupus erythematosus & 0.032 \\
\hline \multicolumn{3}{|l|}{ Green module } \\
\hline hsa04610 & Complement and coagulation cascades & 0.0028 \\
\hline hsa05133 & Pertussis & 0.0132 \\
\hline hsa04010 & MAPK signaling pathway & 0.0394 \\
\hline \multicolumn{3}{|l|}{ Pink module } \\
\hline hsa05322 & Systemic lupus erythematosus & 0.0161 \\
\hline \multicolumn{3}{|l|}{ Purple module } \\
\hline hsa04145 & Phagosome & 0.00051 \\
\hline hsa00380 & Tryptophan metabolism & 0.0024 \\
\hline hsa00340 & Histidine metabolism & 0.0176 \\
\hline hsa04066 & HIF-1 signaling pathway & 0.0176 \\
\hline hsa04640 & Hematopoietic cell lineage & 0.0176 \\
\hline hsa04216 & Ferroptosis & 0.0327 \\
\hline hsa00480 & Glutathione metabolism & 0.0396 \\
\hline hsa04979 & Cholesterol metabolism & 0.0396 \\
\hline \multicolumn{3}{|c|}{ Turquoise module (top 10) } \\
\hline hsa05167 & Kaposi's sarcoma-associated herpesvirus infection & $2.06 \mathrm{E}-05$ \\
\hline hsa05161 & Hepatitis B & $4.52 \mathrm{E}-05$ \\
\hline hsa05162 & Measles & 0.00013 \\
\hline hsa05164 & Influenza A & 0.00013 \\
\hline hsa05321 & Inflammatory bowel disease (IBD) & 0.00024 \\
\hline hsa05140 & Leishmaniasis & 0.00042 \\
\hline hsa04620 & Toll-like receptor signaling pathway & 0.00045 \\
\hline hsa04012 & ErbB signaling pathway & 0.00088 \\
\hline hsa04660 & T cell receptor signaling pathway & 0.0019 \\
\hline hsa04917 & Prolactin signaling pathway & 0.0019 \\
\hline \multicolumn{3}{|l|}{ Yellow module } \\
\hline hsa05340 & Primary immunodeficiency & 4.86E-05 \\
\hline hsa04060 & Cytokine-cytokine receptor interaction & 0.00079 \\
\hline hsa04662 & B cell receptor signaling pathway & 0.00079 \\
\hline hsa04640 & Hematopoietic cell lineage & 0.0018 \\
\hline hsa04650 & Natural killer cell mediated cytotoxicity & 0.006 \\
\hline hsa04659 & Th17 cell differentiation & 0.0143 \\
\hline hsa04660 & T cell receptor signaling pathway & 0.0143 \\
\hline
\end{tabular}


Table 5 (continued)

\begin{tabular}{lll}
\hline KEGG term ID & Term description & FDR \\
\hline hsa04514 & Cell adhesion molecules (CAMs) & 0.0455 \\
hsa04658 & Th1 and Th2 cell differentiation & 0.0493 \\
\hline
\end{tabular}
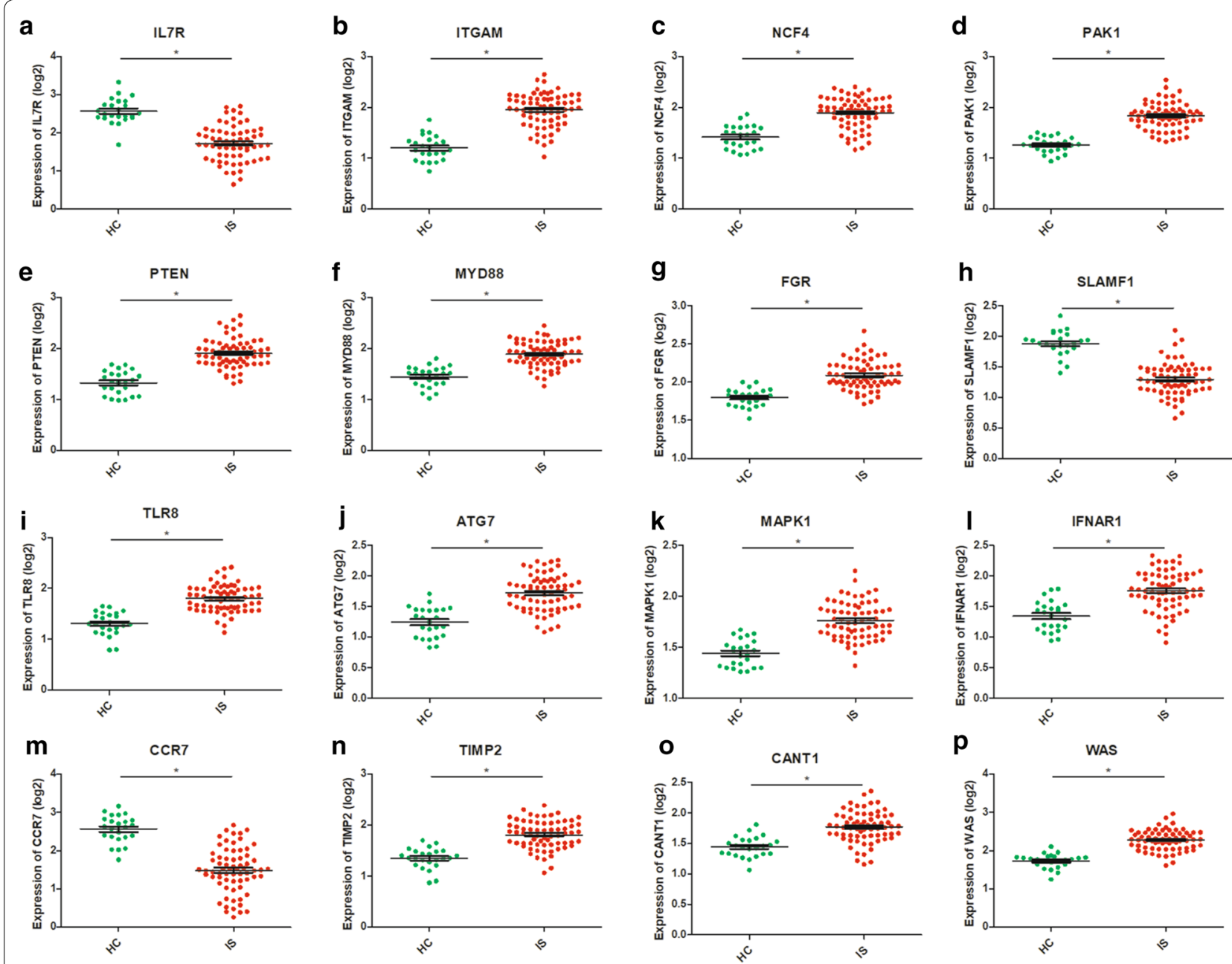

Fig. 3 Verification using GSE37587 dataset. Green: healthy control samples; red: ischemic stroke samples. Statistically significant was considered as the $p$-value < 0.05. a-p Verification of the expression of IL-7R, ITGAM, NCF4, PAK1, PTEN, MYD88, FGR, SLAMF1,TLR8, ATG7, MAPK1, IFNAR1, CCR7, TIMP2, CANT1 and WAS, respectively, in the HC and IS group of validation cohort

two comparison group samples showed individual differences, and the cluster analysis of infiltrated immune cells in disease and normal data is an important means for discovering pathological processes and immunoregulatory mechanisms (Fig. 4b). As shown in Fig. 4c, the proportions of different infiltrated immune cell subpopulations were weakly to moderately correlated. For instance, the correlation of neutrophils and $\mathrm{CD} 8^{+} \mathrm{T}$ cells is 0.63 , and the correlation of $\mathrm{CD}^{+} \mathrm{T}$ cells and naïve $\mathrm{CD}^{+} \mathrm{T}$ cells is 0.61 and so on. Compared with normal samples, stroke samples generally contained a higher proportion for $\gamma \delta$ T cells $(\mathrm{p}=0.044)$, macrophages $M 1(p=0.028)$, macrophages $M 2(p=0.012)$ and neutrophils $(p<0.001)$, whereas the resting dendritic cells $(\mathrm{p}=0.001)$ and eosinophils $(\mathrm{p}=0.025)$ fraction was relatively lower (Fig. 4d).

For further analysis the correlation between hub genes expression and proportions of neutrophils infiltrating, 


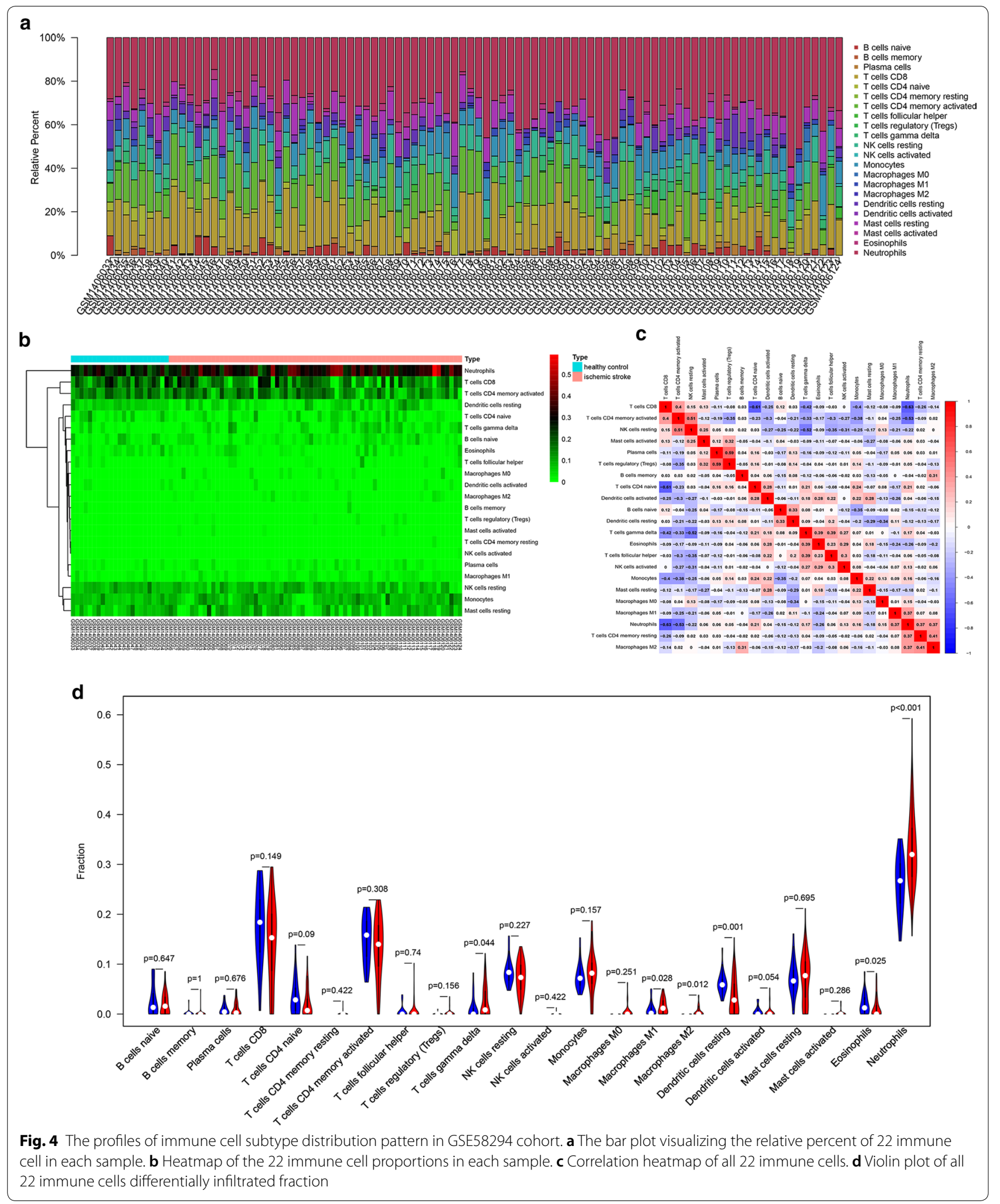


we divided the sample into high proportion neutrophils infiltrated group and low proportion neutrophils infiltrated group. The results of Wilcoxon test indicated that the expression of hub genes were significantly correlated with proportions of neutrophils infiltrating (TLR8, $\mathrm{p}=2.99 \mathrm{E}-09$; MAPK $1, \mathrm{p}=7.05 \mathrm{E}-08$; PAK1, $\quad \mathrm{p}=1.83 \mathrm{E}-05 ; \quad$ PTEN, $\quad \mathrm{p}=4.67 \mathrm{E}-11$; IFNAR1,$\quad \mathrm{p}=4.11 \mathrm{E}-07 ; \quad$ CCR7, $\quad \mathrm{p}=0.010322089$; SLAMF1, $\quad \mathrm{p}=3.23 \mathrm{E}-05 ; \quad$ IL7R, $\quad \mathrm{p}=0.00816272$; TIMP2, $\quad \mathrm{p}=6.54 \mathrm{E}-07 ; \quad$ MYD88, $\quad \mathrm{p}=1.35 \mathrm{E}-07$; CANT1, $\quad p=3.96 \mathrm{E}-06 ; \quad$ ATG7, $\quad \mathrm{p}=5.80 \mathrm{E}-05$; NCF4, $p=3.24 \mathrm{E}-07$; ITGAM, $\mathrm{p}=2.21 \mathrm{E}-07$; WAS, $\mathrm{p}=0.000121197 ; \quad F G R, \mathrm{p}=2.51 \mathrm{E}-09)$, as shown in Fig. $5 \mathrm{a}-\mathrm{p}$. The proportion of neutrophil distributed in the peripheral blood of stroke patients increased, in which the gene expression of IL7R, SLAMF1 and CCR7 were low, and vice versa. The results are consistent with our studies shown in Fig. 3a-p and Fig. 4d.

\section{Verification in the clinical samples}

To further validate the results of the microarray analysis, we examined the expression of the 16 real hub genes which were dysregulated in IS by the qRT-PCR with the blood samples from fifteen IS patients and another fifteen healthy control individuals. And we found that there were 12 genes dysregulated in validation blood samples. Unpaired $t$ test with Welch's correction was used to make statistics of the data shown in Fig. 6a-l. Finally, these genes in both the GSE37587 dataset and our clinical blood samples were overlapped (Fig. 6m), among these ITGAM, NFC4, PTEN, MYD88, FGR, TIMP2 and CANT1 were upregulated in IS samples and IL-7R, SLAMF1 and CCR7 were downregulated in IS samples. Taken together, despite four hub genes did not show significant, our experiment results were generally consistent with the trends of bioinformatics analysis results. And the results of blood routine examination indicated that the percent of neutrophil granulocyte was increased in acute ischemic stroke patients (Fig. 6n). The expansion of harmful neutrophils subsets associated with disease severity may play an important role by promoting systemic inflammation and disruption of the blood-brain barrier.

\section{Discussion}

Ischemic stroke is a severe disease with high incidence and fatality rate and one of the main causes of adult lifetime disability, which leave heavy pressure and burden
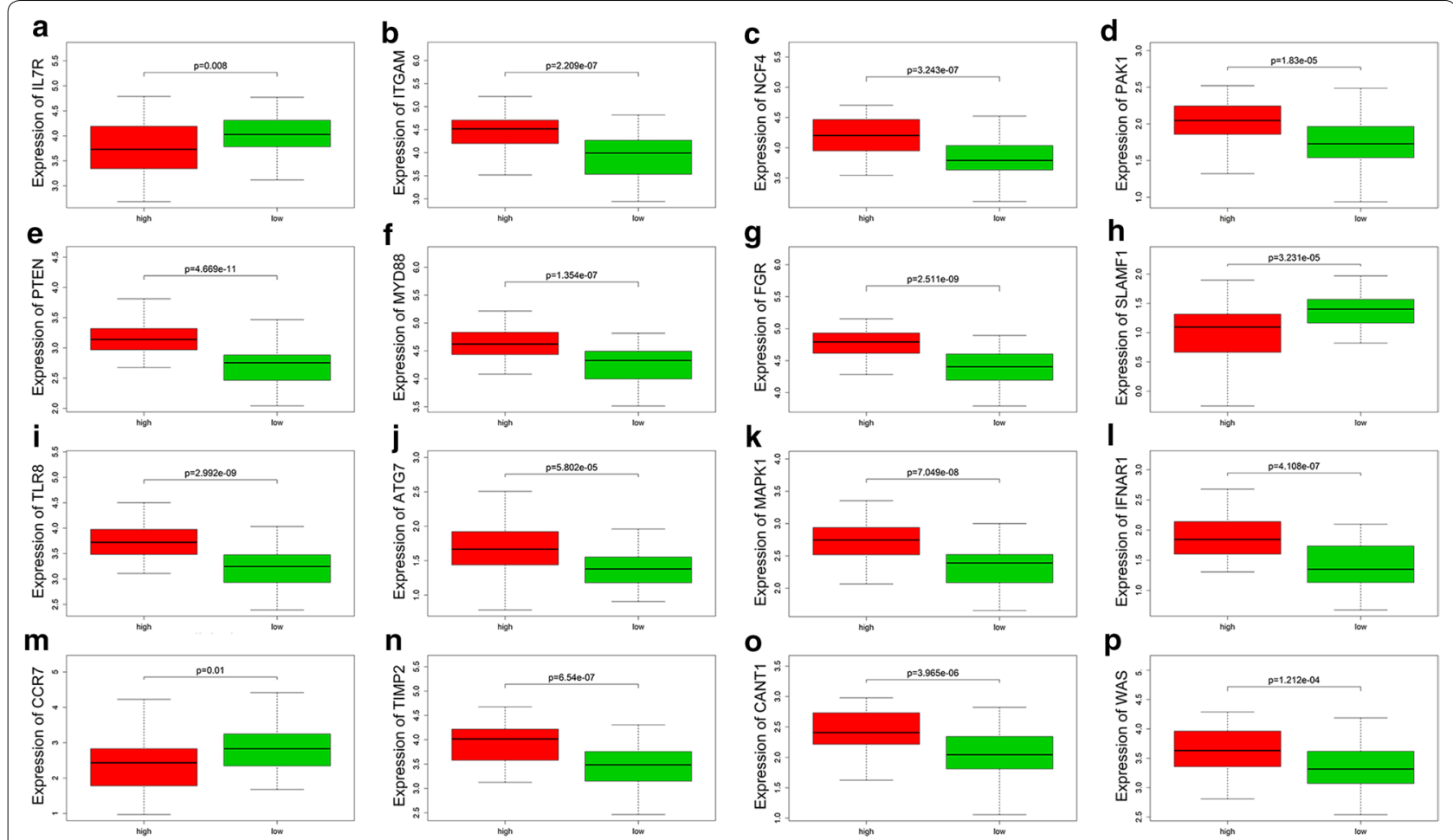
green bar: samples with a low proportion of neutrophils. Statistically significant was considered as the $p$-value $<0.05$. a-p Correlation analysis of the proportion of neutrophils and expression of IL-7R, ITGAM, NCF4, PAK1, PTEN, MYD88, FGR, SLAMF1, TLR8, ATG7, MAPK1, IFNAR1, CCR7, TIMP2, CANT1 and WAS, respectively 


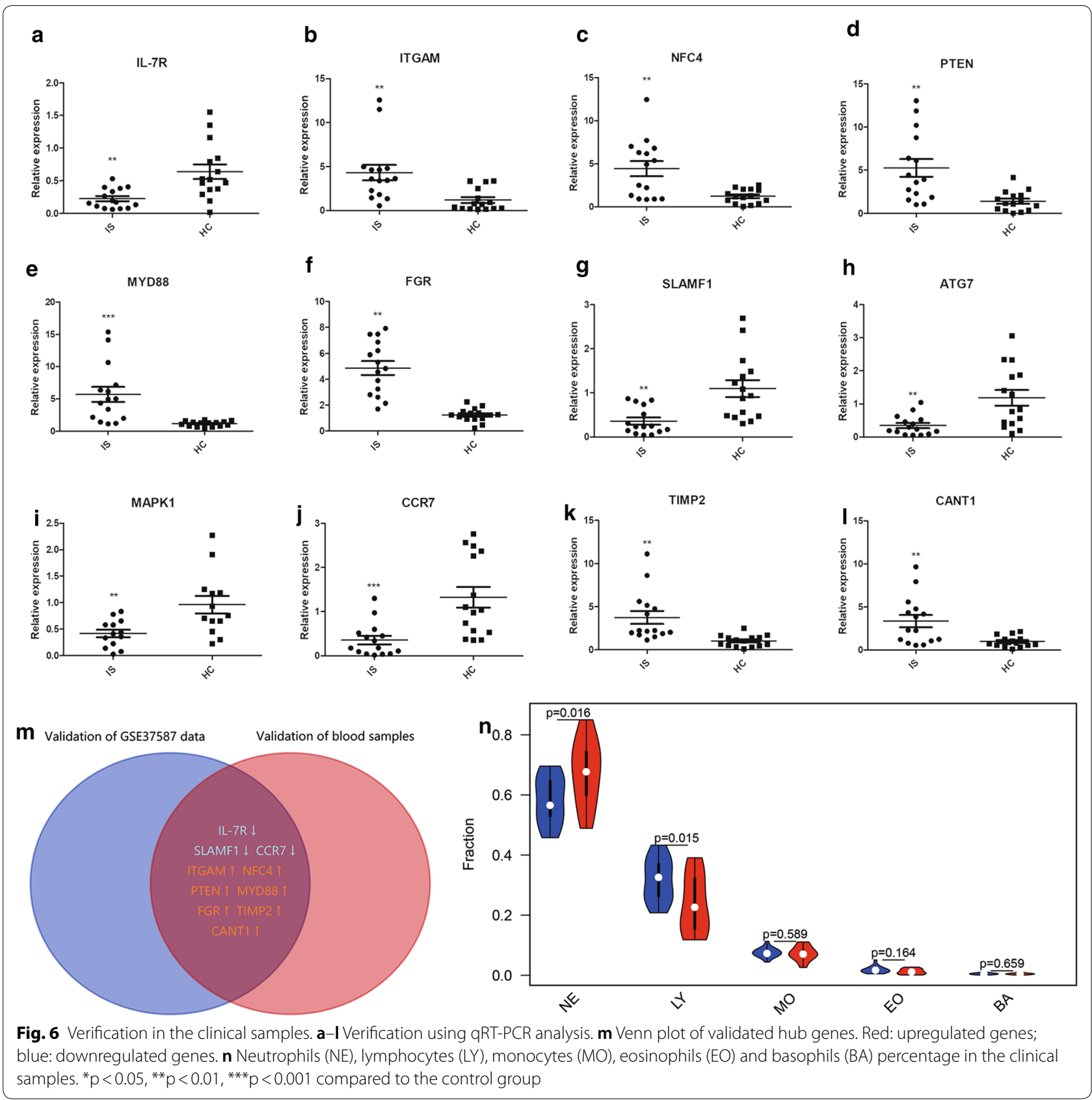

to patients' families and society. Therefore, researchers have realized that it is very urgent to improve the early preclinical diagnosis and treatment level. In this study, WGCNA and CIBERSORT algorithms were used to explore the pathological process and marker genes in peripheral blood samples of ischemic stroke. After data preprocessing and weighted gene network construction, the modules were associated with feature and function enrichment analysis. GO and KEGG analysis identified black, blue, green, pink, purple, turquoise and yellow module as immune-related modules. According to the module recognition heatmap and scatter diagrams, the modules related to stroke onset were black, blue, green, pink, purple, turquoise and yellow module $(\mathrm{p}<0.05)$ (Fig. $2 \mathrm{~b}-\mathrm{i}$ ). By taking overlap, the 7 hub modules are obtained for further analysis. Finally, we found out 16 hub genes distributed across 3 modules. There are TLR8, PTEN and IFNAR1 in turquoise module, CCR7, IL7R and SLAMF1 in yellow module, TIMP2, CANT1, PAK1, 
ATG7, NCF4, ITGAM, WAS, MYD88, MAPK1 and FGR in blue module.

Tumor suppressor PTEN is highly expressed in neurons. Ischemic stroke induced neuronal PTEN degradation and led to cognitive impairment [10]. However, PTEN has been reported to be inhibited by many microRNA (miRNA) and performed neuroprotective effect against ischemic stroke in experimental models. miR-103 can target PTEN and downregulate its expression, which depletion restrains the progression of atherosclerosis through blocking PTEN-mediated MAPK signaling [11]. Another data demonstrates that miR-130a prevented cerebral ischemia/reperfusion damage against ischemic stroke by mediating the PTEN/PI3K/AKT pathway [12]. On the other side, long non-coding RNA (lncRNA) SETD5-AS1 participates in the development of ischemic stroke by activating PTEN and inhibiting PI3K/AKT pathway [13].

Innate immunity plays an important role in brain ischemic injury, toll-like receptors are innate immunity receptors that activate inflammation and adaptive immunity. Many studies have focused on the role of TLR2 and TLR4 in brain damage caused by ischemic stroke. However, the role of TLR8 in ischemic stroke is relatively rare. Firstly, increasing TLR8 expression was found associated with greater inflammatory response, bigger infarct volumes and poor outcomes [14]. Then, Tang and colleagues found that TLR8 could promote neuronal apoptosis and $\mathrm{T}$ cell-mediated inflammation following stroke [15]. Recently, it was reported that TLR8 gene rs3764880 polymorphism might be associated with susceptibility and involved in the inflammatory response and lipid metabolism of ischemic stroke in southern Chinese Han population [16].

Type 1 IFN-stimulated genes reaction in the microglia exposed to ischemia/reperfusion depends on innate immune receptors including TLR4 and IFNAR1 [17], which played a deleterious role in the outcome after stroke [18]. Microglia-targeted IFNAR1 knockdown demonstrated that interferon signaling specifically in microglia is essential for the protection effect of ischemic preconditioning [19]. Furthermore, the IFNAR1 knockdown cells were protected against oxygen-glucosedeprivation induced neuro-inflammation with reduced pro-apoptotic cleaved caspase-3 levels, compared to negative control cells [20].

Our results indicated that there is a negative correlation between the expression of genes in the yellow module and the occurrence of ischemic stroke. Chemokine receptor CCR7 comes from a wide range of sources in the body, including peripheral blood neutrophils, macrophages and glial cells of CNS. Ischemic stroke plays a bipolar role in the peripheral immune response. Peripheral leukocyte activation occurs early, followed by severe immunosuppression, characterized by splenic atrophy and reduced expression of cytokines and chemokines [21]. Peripheral immune cells such as T cells migrate to the brain and exacerbate the developing infarct [22]. Studies have shown that the relative mRNA expression of CCR7 in peripheral blood of patients with traumatic brain injury within $24 \mathrm{~h}$ after onset was significantly reduced, compared with the normal control group [23], which was consistent with our experimental results.

Costimulatory molecule and microbial sensor SLAMF1 (also known as CD150) initiates signal transduction pathways in a variety of immune cells [24]. SLAMF1 is overexpressed on the T cell, B cell and their subgroups of Systemic lupus erythematosus (SLE) patients [25, 26]. Expression of SLAMF1 was significantly increased in peripheral blood CD4+, T helper 17, and CD19+ B cells from autoimmune thyroid disease patients [27]. In addition, compared with the healthy control group, SLAMF1 was significantly down-regulated in patients with acute myocardial infarction (AMI) [28]. Evidence has been accumulating that SLAM family members are potential targets for inflammatory and autoimmune diseases [29]. Bacille Calmette-Guérin (BCG) infection significantly upregulated SLAMF1, which enhanced inflammatory response by activating the NF- $k B$ signaling pathway [30]. Yurchenko and colleagues reported that SLAMF1 is a new target for modulation of TLR4-TRAM-TRIF inflammatory signaling in human cells [24]. However, the dysfunction of SLAMF1 in ischemic stroke has not been reported before. In this study, we found that SLAMF1 was downregulated in the IS patient samples which indicate that SLAMF1 may be a potential target for medical intervention in patients with ischemic stroke. And its role in immune regulation needs to be further elucidated.

IL7R widely express in immunity system and play critical roles in immune cell development and immune system homeostasis. Studies showed that the IL7R allele polymorphism was associated with an increased risk of multiple sclerosis (MS) [31], and there was a significant association between the IL7R promoter polymorphisms and the age of onset of MS [32]. Furthermore, the latest evidence shows that the lymphoid-associated interleukin 7 receptor (IL7R) regulates tissue-resident macrophage development [33]. However, the association between IL7R expression and IS onset has not been reported before. In the present study, it was found that IL7R was low expression in IS cohorts. Larger-scale studies of populations and in-depth researches are needed to explore the roles played by the IL7R during the pathogenesis of IS.

Our results indicate that the expression of TIMP-2 and MAPK1 upregulated in IS, which are consistent 
with the finding of Liu NN and colleagues [34]. In addition, their research illustrates that the TIMP2-dependent MAPK pathway could be negatively regulated by miR-410, which exert neuroprotective role and against oxidative stress-induced apoptosis after IS in mice models. On the other hand, another study indicated that overexpressing TIMP2 and TIMP1 in rat middle cerebral artery occlusion (MCAO) model 3 days before occlusion could receive the larger beneficial effect such as smaller infarct size, and better motor function recovery than autologous bone marrow cells alone or in combination with drugs [35].

CANT1 was reported been associated with chondrodysplasia [36], clear cell renal cell carcinoma [37], with no evidence for an association with IS.

Mitsios and colleagues reported for the first time an upregulated expression of PAK1 in human after IS and rat brain samples following MCAO [38]. PAK1, as a major cyclin-dependent kinase 5 (Cdk5) substrate and target, could be hyperphosphorylated by p35/Cdk5, leading to the downregulation and activity inhibition of PAK1 [39]. PAK1 involved in many actin dynamics pathways, and its expression dysregulation might cause loss of synapses, cognitive deficits, and impaired motor functions.

Lee and colleagues performed genome-wide association studies (GWAS) for small-vessel occlusion (SVO) stroke and the results showed that three single nucleotide polymorphisms in ATG7 were associated with SVO stroke [40]. Cerebral endothelial ATG7 was reported that could modulate pro-inflammatory cytokines expression and lead to brain ischemia/reperfusion injury during stroke [41]. Furthermore, the previous study of our research team [42] has been reported that KCNQ1OT1 promotes autophagy by regulating miR-200a/FOXO3/ ATG7 pathway in cerebral ischemic stroke.

NADPH-complex component p40-phox subunit (encoded by NCF4), a key factor in biochemical pathways and the innate immune response. Due to its important role in the innate immune response, the gene polymorphism of NCF4 has been reported to be involved in chronic granulomatous disease [43, 44] and increased the risk of colorectal cancer [45]. Some researches demonstrated that NCF4 might be a potential diagnostic biomarker or a regulatory target for acute myocardial infraction (AMI) [46] and coronary artery disease [47]. We suspect that NCF4 act on similar modes and approaches in cerebral vascular diseases compared to those of the cardiovascular diseases.

ITGAM (also known as CD11b) is predominantly expressed in monocytes, macrophages and granulocytes, involved in various adhesive interactions and mediating the uptake of complement-coated particles. ITGAM genetic variations was reported be associated with susceptibility to SLE [48]. As far as we know, during ischemic stroke CD11b was strongly expressed on the activated macrophage/microglia and infiltrating leukocytes, reflecting the clinical severity of inflammatory response in the brain [49].

To the best of our knowledge, TLR4/MyD88/MAPK/ $\mathrm{NF}-\mathrm{KB}$ signal pathway was one of the classical pathways in ischemic stroke-induced inflammation. Many drugs [50,51] and treatments [52] could inhibit the TLR4-mediated inflammatory responses and decrease proinflammatory cytokine release through the MyD88dependent signaling pathway. MyD88-dependent signaling contributes to the inflammatory responses induced by cerebral ischemia/reperfusion [53].

Some studies indicate that the inflammatory properties of circulating neutrophils increase during acute ischemic stroke [49], which similar to our research results (Fig. 4a). The expansion of harmful neutrophils subsets associated with disease severity may play an important role by promoting systemic inflammation and disruption of the blood-brain barrier. We speculated that these mRNA signal of the analyzed genes comes mainly form neutrophils (related not only to neutrophils number increased, but also to the neutrophils activated). New therapeutic approaches of stroke by rebalancing the neutrophil subset homeostasis may become potential targeted therapies. In addition, modulating macrophages/microglia [54], or Th17/ $\gamma \delta$ T cells [55] subsets with biologics after stroke have become a topic of interest in recent years, and the discovery of new drugs related to $\mathrm{T}$ cell subsets or macrophages/microglia polarization may enable the realization of stroke targeted therapies.

\section{Conclusion}

In conclusion, our study is the first to integrate most comprehensive microarray samples of ischemic stroke for WGCNA. In this study, we demonstrated the 7 immune related gene expression modules and 16 hub genes, in which the SLAMF1, IL7R and NCF4 may be novel candidate biomarkers or therapeutic targets and never reported be related to IS before. Our findings may provide valuable reference for further pathogenesis mechanism elucidation of IS. Furthermore, these hub genes and neutrophils may become important biological targets in the drug screening and drug designing. 


\section{Supplementary information}

Supplementary information accompanies this paper at https://doi. org/10.1186/s12967-020-02463-0.

Additional file 1: Figure S1. A workflow of the analysis procedure. HC, healthy control; IS, ischemic stroke; GO, gene oncology; KEGG, Kyoto encyclopedia of genes and genomes.

Additional file 2: Table S1. Sequences of primers used for qPCR. Table S2. Clinical characteristics of validation samples.

Additional file 3. The expression matrices of total 1082 DEGs in training set.

Additional file 4: Figure S2. Samples clustering and identification of differentially expressed genes (DEGs) in IS samples. (A) Samples clustering of total 195 samples to detect outliers. (B) Re-clustering of 188 samples: sample dendrogram and trait heatmap. The clustering was based on the expression data of DEGs between healthy controls $(\mathrm{HC})$ and ischemic stroke (IS) patients. In disease state, white means HC and red means IS.

Additional file 5: Figure S3. Determination of soft-thresholding power in the WGCNA. (A) Analysis of the scale-free index for a set of softthresholding powers ( $\beta$ ). (B) Analysis of the mean connectivity for a set of soft-thresholding powers. (C) Histogram of connectivity distribution when $\beta=8$. (D) Checking the scale free topology when $\beta=8$.

Additional file 6: Figure S5. Scatter diagrams for module membership vs. gene significance of disease state in brown(A), magenta(B), red(C) and grey(D) module

Additional file 7: Figure S4. PPI network in different modules. (A) Yellow module. (B) Black module. (C) Blue module. (D) Turquoise module. The yellow nodes represent the hub genes (connectivity degree $>8$ ) in each module.

Additional file 8: Figure S6. PPI networks of purple(A), pink(B) and green (C) module.

\section{Abbreviations}

IS: Ischemic stroke; WGCNA: Weighted gene co-expression network analysis; GEO: Gene Expression Omnibus; GO: Gene oncology; KEGG: Kyoto encyclopedia of genes and genomes; STRING: Search Tool for the retrieval of Interacting Genes/Proteins; DEGs: Differentially expressed genes; PPI: Protein-protein interaction; miRNA: microRNA; IncRNA: Long non-coding RNA; SLE: Systemic lupus erythematosus; MS: Multiple sclerosis; MCAO: Middle cerebral artery occlusion; GWAS: Genome-wide association studies; AMI: Acute myocardial infraction.

\section{Acknowledgements}

None.

\section{Authors' contributions}

ZL wrote the article, created the figures, performed the verification experiments and provided critical revision. YC collected the clinical samples. JF and YG provided critical revisions and conceptual support. All authors read and approved the final manuscript.

\section{Funding}

The laboratory reagents for PCR test were supported financially by the National Natural Science Foundation of China (grant number 81771271) to Juan Feng.

\section{Availability of data and materials}

The datasets during and/or analyzed during the current study available from the corresponding author on reasonable request.

\section{Ethics approval and consent to participate}

This study was approved by the Ethics Committee of Shengjing Hospital, China Medical University (No. 2017PS161K). Informed consent was obtained from the patients for publication of this manuscript.

\section{Consent for publication}

Not applicable.

\section{Competing interests}

The authors declare that they have no competing interests.

Received: 12 October 2019 Accepted: 28 July 2020

Published online: 03 August 2020

\section{References}

1. Hasan TF, Rabinstein AA, Middlebrooks EH, Haranhalli N, Silliman SL, Meschia JF, Tawk RG. Diagnosis and management of acute ischemic stroke. Mayo Clin Proc. 2018;93:523-38.

2. Krishnan S, Lawrence CB. Old dog new tricks; revisiting how stroke modulates the systemic immune landscape. Front Neurol. 2019;10:718.

3. Jayaraj RL, Azimullah S, Beiram R, Jalal FY, Rosenberg GA. Neuroinflammation: friend and foe for ischemic stroke. J Neuroinflamm. 2019;16:142.

4. Javidi E, Magnus T. Autoimmunity after ischemic stroke and brain injury. Front Immunol. 2019;10:686.

5. Miller JA, Woltjer RL, Goodenbour JM, Horvath S, Geschwind DH. Genes and pathways underlying regional and cell type changes in Alzheimer's disease. Genome Med. 2013:5:48.

6. Radulescu E, Jaffe AE, Straub RE, Chen Q, Shin JH, Hyde TM, Kleinman JE, Weinberger DR. Identification and prioritization of gene sets associated with schizophrenia risk by co-expression network analysis in human brain. Mol Psychiatry. 2018;25:791-804.

7. Ali HR, Chlon L, Pharoah PD, Markowetz F, Caldas C. Patterns of immune infiltration in breast cancer and their clinical implications: a gene-expression-based retrospective study. PLoS Med. 2016:13:e1002194.

8. Xiong Y, Wang K, Zhou H, Peng L, You W, Fu Z. Profiles of immune infiltration in colorectal cancer and their clinical significant: a gene expressionbased study. Cancer Med. 2018;7:4496-508.

9. Pearson GS. Reviewing manuscripts with problematic language issues. J Am Psychiatr Nurses Assoc. 2019;25:251-2.

10. Li W, Huang R, Chen Z, Yan LJ, Simpkins JW, Yang SH. PTEN degradation after ischemic stroke: a double-edged sword. Neuroscience. 2014;274:153-61.

11. Jiang $L$, Qiao $Y$, Wang Z, Ma X, Wang H, Li J. Inhibition of microRNA-103 attenuates inflammation and endoplasmic reticulum stress in atherosclerosis through disrupting the PTEN-mediated MAPK signaling. J Cell Physiol. 2019;235:380-93

12. Zheng T, Shi Y, Zhang J, Peng J, Zhang X, Chen K, Chen Y, Liu L. MiR-130a exerts neuroprotective effects against ischemic stroke through PTEN/ PI3K/AKT pathway. Biomed Pharmacother. 2019;117:109117.

13. Miao SY, Miao SM, Cui RT, Yu AL, Miao ZJ. SETD5-AS1 stimulates neuron death in stroke via promoting PTEN expression. Eur Rev Med Pharmacol Sci. 2018;22:6035-41.

14. Brea D, Sobrino T, Rodriguez-Yanez M, Ramos-Cabrer P, Agulla J, Rodriguez-Gonzalez R, Campos F, Blanco M, Castillo J. Toll-like receptors 7 and 8 expression is associated with poor outcome and greater inflammatory response in acute ischemic stroke. Clin Immunol. 2011:139:193-8.

15. Tang SC, Yeh SJ, Li YI, Wang YC, Baik SH, Santro T, Widiapradja A, Manzanero S, Sobey CG, Jo DG, et al. Evidence for a detrimental role of TLR8 in ischemic stroke. Exp Neurol. 2013;250:341-7.

16. Gu L, Zhou J, Tan J, Yang J, Shen T, Jiang H, Tang Q. Association of TLR8 gene rs3764880 polymorphisms with susceptibility and lipid metabolism- and inflammation response-related quantitative traits of ischemic stroke in southern Chinese Han male population. J Neurol Sci. 2016;370:94-9.

17. McDonough A, Lee RV, Noor S, Lee C, Le T, lorga M, Phillips JLH, Murphy S, Moller T, Weinstein JR. Ischemia/reperfusion induces interferon-stimulated gene expression in microglia. J Neurosci. 2017;37:8292-308.

18. Zhang M, Downes CE, Wong CHY, Brody KM, Guio-Agulair PL, Gould J, Ates R, Hertzog PJ, Taylor JM, Crack PJ. Type-l interferon signalling through IFNAR1 plays a deleterious role in the outcome after stroke. Neurochem Int. 2017:108:472-80.

19. Hamner MA, Ye Z, Lee RV, Colman JR, Le T, Gong DC, Ransom BR, Weinstein JR. Ischemic preconditioning in white matter: magnitude and mechanism. J Neurosci. 2015:35:15599-611. 
20. Minter MR, Zhang M, Ates RC, Taylor JM, Crack PJ. Type-1 interferons contribute to oxygen glucose deprivation induced neuro-inflammation in BE(2)M17 human neuroblastoma cells. J Neuroinflamm. 2014;11:43.

21. Zhang B, Subramanian S, Dziennis S, Jia J, Uchida M, Akiyoshi K, Migliati $E$, Lewis AD, Vandenbark AA, Offner $H$, Hurn PD. Estradiol and G1 reduce infarct size and improve immunosuppression after experimental stroke. J Immunol. 2010;184:4087-94.

22. Dziennis S, Mader S, Akiyoshi K, Ren X, Ayala P, Burrows GG, Vandenbark AA, Herson PS, Hurn PD, Offner HA. Therapy with recombinant T-cell receptor ligand reduces infarct size and infiltrating inflammatory cells in brain after middle cerebral artery occlusion in mice. Metab Brain Dis. 2011;26:123-33.

23. Petrone $A B$, Gionis V, Giersch R, Barr TL. Immune biomarkers for the diagnosis of mild traumatic brain injury. NeuroRehabilitation. 2017:40:501-8.

24. Yurchenko M, Skjesol A, Ryan L, Richard GM, Kandasamy RK, Wang N, Terhorst C, Husebye H, Espevik T. SLAMF1 is required for TLR4-mediated TRAM-TRIF-dependent signaling in human macrophages. J Cell Biol. 2018:217:1411-29.

25. Karampetsou MP, Comte D, Kis-Toth K, Kyttaris VC, Tsokos GC. Expression patterns of signaling lymphocytic activation molecule family members in peripheral blood mononuclear cell subsets in patients with systemic lupus erythematosus. PLoS ONE. 2017;12:e0186073.

26. Karampetsou MP, Comte D, Suarez-Fueyo A, Katsuyama E, Yoshida N, Kono M, Kyttaris VC, Tsokos GC. Signaling lymphocytic activation molecule family member 1 engagement inhibits T cell-B cell interaction and diminishes interleukin-6 production and plasmablast differentiation in systemic lupus erythematosus. Arthr Rheumatol. 2019;71:99-108.

27. Vitales-Noyola M, Ramos-Levi AM, Serrano-Somavilla A, Martinez-Hernandez R, Sampedro-Nunez M, Di Pasquale C, Gonzalez-Amaro R, Marazuela M. Expression and function of the costimulatory receptor SLAMF1 is altered in lymphocytes from patients with autoimmune thyroiditis. J Clin Endocrinol Metab. 2017;102:672-80.

28. Yan W, Song H, Jiang J, Xu W, Gong Z, Duan Q, Li C, Xie Y, Wang L. Characteristics of $B$ cellassociated gene expression in patients with coronary artery disease. Mol Med Rep. 2016;13:4113-21.

29. Dragovich MA, Mor A. The SLAM family receptors: potential therapeutic targets for inflammatory and autoimmune diseases. Autoimmun Rev. 2018;17:674-82

30. Song T, Dong C, Xiong S. Signaling lymphocyte-activation molecule SLAMF1 augments mycobacteria BCG-induced inflammatory response and facilitates bacterial clearance. Int J Med Microbiol. 2015;305:572-80

31. Wu S, Liu Q, Zhu JM, Wang MR, Li J, Sun MG. Association between the IL7R T244I polymorphism and multiple sclerosis risk: a meta analysis. Neurol Sci. 2016:37:1467-74.

32. Simsek H, Geckin H, Sensoz NP, List EO, Arman A. Association between IL7R promoter polymorphisms and multiple sclerosis in turkish population. J Mol Neurosci. 2019;67:38-47.

33. Leung $G A$, Cool $T$, Valencia $C H$, Worthington $A$, Beaudin AE, Forsberg EC The lymphoid-associated interleukin 7 receptor (IL7R) regulates tissueresident macrophage development. Development. 2019;146:dev176180.

34. Liu NN, Dong ZL, Han LL. MicroRNA-410 inhibition of the TIMP2dependent MAPK pathway confers neuroprotection against oxidative stress-induced apoptosis after ischemic stroke in mice. Brain Res Bull. 2018;143:45-57

35. Baker AH, Sica V, Work LM, Williams-Ignarro S, de Nigris F, Lerman LO, Casamassimi A, Lanza A, Schiano C, Rienzo M, et al. Brain protection using autologous bone marrow cell, metalloproteinase inhibitors, and metabolic treatment in cerebral ischemia. Proc Natl Acad Sci U S A. 2007; 104:3597-602.

36. Paganini C, Monti L, Costantini R, Besio R, Lecci S, Biggiogera M, Tian K, Schwartz JM, Huber C, Cormier-Daire V, et al. Calcium activated nucleotidase 1 (CANT1) is critical for glycosaminoglycan biosynthesis in cartilage and endochondral ossification. Matrix Biol. 2019:81:70-90.

37. Liu X, Yang Z, Luo X, Luo J, Fu W, Fang Z, Xia D, Li L, Xu J. Calciumactivated nucleotidase 1 silencing inhibits proliferation, migration, and invasion in human clear cell renal cell carcinoma. J Cell Physiol. 2019;234:22635-47
38. Mitsios N, Saka M, Krupinski J, Pennucci R, Sanfeliu C, Wang Q, Rubio F, Gaffney J, Kumar P, Kumar S, et al. A microarray study of gene and protein regulation in human and rat brain following middle cerebral artery occlusion. BMC Neurosci. 2007;8:93.

39. Shah K, Rossie S. Tale of the good and the bad Cdk5: remodeling of the actin cytoskeleton in the brain. Mol Neurobiol. 2018;55:3426-38.

40. Lee TH, Ko TM, Chen CH, Chang YJ, Lu LS, Chang CH, Huang KL, Chang TY, Lee JD, Chang KC, et al. A genome-wide association study links smallvessel ischemic stroke to autophagy. Sci Rep. 2017:7:15229.

41. Wang HJ, Wei JY, Liu DX, Zhuang SF, Li Y, Liu H, Ban M, Fang WG, Cao L, Zhao WD, Chen YH. Endothelial Atg7 deficiency ameliorates acute cerebral injury induced by ischemia/reperfusion. Front Neurol. 2018;9:998.

42. Yu S, Yu M, He X, Wen L, Bu Z, Feng J. KCNQ1OT1 promotes autophagy by regulating miR-200a/FOXO3/ATG7 pathway in cerebral ischemic stroke. Aging Cell. 2019;18:e12940.

43. Chiriaco M, Salfa I, Di Matteo G, Rossi P, Finocchi A. Chronic granulomatous disease: clinical, molecular, and therapeutic aspects. Pediatr Allergy Immunol. 2016;27:242-53.

44. Wu PB, Dai JF, Wang Q, Zhang G, Tan SY, Li M, Ye HL. Association between NCF4 rs4821544T/C polymorphism and inflammatory bowel disease risk in Caucasian: a meta-analysis. Inflamm Res. 2015;64:825-31.

45. Ryan BM, Zanetti KA, Robles Al, Schetter AJ, Goodman J, Hayes RB, Huang WY, Gunter MJ, Yeager M, Burdette L, et al. Germline variation in NCF4, an innate immunity gene, is associated with an increased risk of colorectal cancer. Int J Cancer. 2014;134:1399-407.

46. Chen J, Yu L, Zhang S, Chen X. Network analysis-based approach for exploring the potential diagnostic biomarkers of acute myocardial infarction. Front Physiol. 2016;7:615

47. Wang Y, Liu T, Liu Y, Chen J, Xin B, Wu M, Cui W. Coronary artery disease associated specific modules and feature genes revealed by integrative methods of WGCNA, MetaDE and machine learning. Gene. 2019;710:122-30

48. Ho RC, Ong H, Thiaghu C, Lu Y, Ho CS, Zhang MW. Genetic variants that are associated with neuropsychiatric systemic lupus erythematosus. J Rheumatol. 2016:43:541-51.

49. Weisenburger-Lile D, Dong Y, Yger M, Weisenburger G, Polara GF, Chaigneau T, Ochoa RZ, Marro B, Lapergue B, Alamowitch S, Elbim C. Harmful neutrophil subsets in patients with ischemic stroke: association with disease severity. Neurol Neuroimmunol Neuroinflamm. 2019;6:e571.

50. Wu LR, Liu L, Xiong XY, Zhang Q, Wang FX, Gong CX, Zhong Q, Yang YR, Meng ZY, Yang QW. Vinpocetine alleviate cerebral ischemia/reperfusion injury by down-regulating TLR4/MyD88/NF-kappaB signaling. Oncotarget. 2017:8:80315-24.

51. Zhu S, Tang S, Su F. Dioscin inhibits ischemic strokeinduced inflammation through inhibition of the TLR4/MyD88/NFkappaB signaling pathway in a rat model. Mol Med Rep. 2018;17:660-6.

52. Ye Y, Yang Y, Chen C, Li Z, Jia Y, Su X, Wang C, He X. Electroacupuncture improved hippocampal neurogenesis following traumatic brain injury in mice through inhibition of TLR4 signaling pathway. Stem Cells Int. 2017;2017:5841814

53. Ye X, Kong D, Wang J, Ishrat T, Shi H, Ding X, Cui G, Hua F. MyD88 contributes to neuroinflammatory responses induced by cerebral ischemia/ reperfusion in mice. Biochem Biophys Res Commun. 2016:480:69-74.

54. Wang J, Xing H, Wan L, Jiang X, Wang C, Wu Y. Treatment targets for M2 microglia polarization in ischemic stroke. Biomed Pharmacother. 2018;105:518-25

55. Shekhar S, Cunningham MW, Pabbidi MR, Wang S, Booz GW, Fan F. Targeting vascular inflammation in ischemic stroke: recent developments on novel immunomodulatory approaches. Eur J Pharmacol. 2018;833:531-44.

\section{Publisher's Note}

Springer Nature remains neutral with regard to jurisdictional claims in published maps and institutional affiliations. 